\title{
Developing weather forecasting system in Georgia
}

Volume 2 Issue 7 - 2017

\section{Short communication}

Weather regional forecasting is the hard mathematical task especially for Georgian complex relief. The Georgian relief may be characterized by three sharply expressed orographic elements: in north Caucasus, in south - Georgian south uplands and lowland or intermountain depression located between those two risings. This one begins from The Black Sea shore by triangular Kolkheti Lowland and spreads up to eastern Georgia like narrow strip. Between those two uplands small scaled orographic elements can be allocated. Such complicated relief has definite influence on air masses motion in atmosphere lower layers. Mainly west and eastern atmospheric processes prevailed over Georgian territory. Due to complex orographic conditions and influence of the black Sea in Georgia exist most of Earths climatic types, from marine wet subtropical climate in west Georgia and steppe continental climate in east Georgia up to eternal snow and glaciers in high mountain zone of Great Caucasus, and also approximately $40 \%$ of observed landscapes. Current geodynamics and orographic properties of Georgia play an important role in formation of weather various patterns. Such complex relief conditions the formation and evolution of various scaled circulation systems and heterogeneous spatial distribution of meteorological elements. This is verified by the fact, that precipitation annual distribution has diverse type, with sharply expressed spatial in homogeneities. The local circulation systems developed on the background of synoptical processes play significant role in the spatialtemporal distribution of weather determining parameters. For present leading weather research centers develop and run real time global and regional forecasting systems. Based on mentioned modeling system Georgian weather forecasting service calculates main meteorological parameters on high resolution grid. Instead of abovesaid operational service still needs creation of such methods that will be able to describe with high spatial-temporal decision and high quality of adequacy. For short-term operational forecasting the use of confined area models became available in several national meteorological services. The range of those models is quite diverse from which special attention deserve regional Mesoscale models also atmosphere dynamical models with artificial boundaries where model variables are defined from coarse value grid from global model outputs. Such models can describe real weather conditions invisible for global models that form in atmosphere small-scale processes.

Local area (space) model structure may be divided in dynamical and physical package. Its configuration for Caucasus region considers relevant adjustment of physical package such as local landscapegeographical properties (including: relief parameters, land use and soil types, soil temperature, plant seasonal distribution and etc.). Dynamical core provides general circulation processes transformation influenced by Caucasus relief and proximity of The Black and Caspian Seas resulting in local weather. The specification of those processes is possible by optimal configuration selection of schemes describing physical processes. Besides ARW provides introduction of higher spatial-temporal resolution horizontal grid that focuses on target subregion and significantly increases model resolution (from $15 \mathrm{~km}$ to 5 $\mathrm{km}$.). Complex relief of Caucasus significantly influences on weather

\author{
Marika Tatishvili \\ Natural and Technogenic Disaster Modeling Department, \\ Georgian Technical University, Georgia
}

\begin{abstract}
Correspondence: Marika Tatishvili, Head of Weather Forecasting, Natural and Technogenic Disaster Modeling Department, Georgian Technical University, Georgia,
\end{abstract} Email tatishvili@yahoo.com

Received: September 21, 2017| Published: November 02, 2017

formation thus relief consideration in model is one of most important assignment. It may be realized by relief parameterization or statistical type or by using both of them.

As calculations show Caucasus orographic is considered at high level in regional model that is proved by atmosphere boundary level pressure forecasting.

a. Clearly fixed local circulation formation in west Georgia during western circulation processes;

b. Also well fixed high pressure area formation in River Mtkvari valley during eastern processes and on the contrary - low pressure area in Rioni River gorge (Kolkheti lowland).

Mentioned facts give possibility to predict with high accuracy the beginning and end of western and eastern incursions on Georgian territory also their intensity, specifically western and eastern wind power in Mtkvari and Rioni rivers. Numerical forecasting models based on complete hydrothermodynamical equations give possibility to detail involve physical factors describing atmospheric phenomena that greatly influenced or sometimes define atmospheric circulation processes. The consideration of those factors in numerical models provides improvement forecasting quality. Realization of weather forecasting issue on confined area is characterized by definite difficulties. Such is the formulation of boundary conditions on the borders of forecasting area. The lack of meteorological data on region borders influenced researches to seek problem solution different ways. By using telescopization or embedded grid method became one of the most effective mean for this. Except boundary conditions telescopization method gives possibility to solve those main issues that are essential for weather forecasting on confined area. Specifically reducing spatial grid step on target area in such way that didn't increase model realization time, also detail describe region orographic features, realize interconnection between largescale, regional and mesoscale meteorological processes using bilateral and unilateral interaction of solutions from different grids. Based on atmospheric 
processes nonstationary mesoscale model ${ }^{1}$ for Georgian territory the peculiarities of mesoscale flows in troposphere under conditions when undisturbed background flow undergo significant transformations and atmospheric circulation regime has been changed by another one. Many efforts and methods have been dedicated to the problem of precipitation formation and convective cloud evolution processes for Georgian conditions. Among others it is remarkable to mention operational thermohydrodynamical convective cloud model created for research of natural and artificial precipitation formation and can be used in weather modification. ${ }^{2}$ In model for crystallization and melting processes have been introduced new parameterization schemes. One of most important precipitation formation microphysical processcoagulation describing integral-differential equations has been analytically solved considering income of cloud particles. The results evidently showed redistribution of ice crystals and rain drops in cloud dispersive medium.

Many researchers and scientists remark that in weather forecasting models detail considering of cloud microphysics would be preferable but as they are micro scale processes usually parameterization schemes are used. The some peculiarities of microstructure of cloud formations have been discussed using quantum disperse forces or Van-Der-Vaals forces that are typical for water particles. To obtain the expression for interaction potential the wave functions of basic and exited states of clusters and dispersion matrix have been introduced describing by virtual photon. It has been turned out that virtual photon interaction causes potential holes and barriers that are decreased by height and width. The isolated long wave quant may be the radiation that is generated throughout observed microphysical processes. The cluster may be presented as multipole system. The multipole is the system composed by couple opposite charges that have definite symmetry type. The simplest is the dipole. If the transition is forbidden in dipole approach it may happen in higher approaches - quadruple (electric) or magnetic dipole. Their probability is approximately 106 times less than dipole. To search out transition probability of cluster from basic state into exciting or virtual one interacting with electromagnetic field the identification of Einstein factors have to be needed. ${ }^{3}$ This new approach can lead research series in different microphysical processes such as phase transitions, electric processes and etc.

\section{Acknowledgments}

None.

\section{Conflicts of interest}

The authors declare there is no conflict of interests.

\section{Funding}

None.

\section{References}

1. M Tatishvili, D Demetrashvili, I Mkurnalidze. Weather forecasting modeling in Georgia. Proceedings of International Scientific Conference Dedicated to Academician I Prangishvili 85 Anniversary "Information and Computer Technologies, Modeling, Control". Georgia: Georgian Technical University; 2015.

2. N Begalishvili, M Tatishvili, G Robitashvili. Investigation of natural and artificial precipitation formation in convective clouds using operational thermohydrodynamical model. Transactions of Institute of Hydrometeorology. 2010;114:44-50.

3. Marika Tatishvili. Energy transformation in clouds according quantum principles. Journal of Environmental Science. 2014;3:7-9. 